22. Sunyaev, R.A. and Zel'dovich, Ya. B. 1972, Comments Ap. Space Phys, 4, p 173. 23. Boynton, P.E., Radford, S.J.E., Schommer, R.A. and Murray, S.S. 1982, Ap. J. 252 , p. 473 .

24. White, S.D.M., Silk, J., Henry, J.P. 1981, Ap. J. Letters 251, L65.

25. Lake, G. and Partrıdge, R.B. 1980, Ap. J. 237, p. 378.

26. Birkinshaw, M., Gull, S.F. and Moffet, A.T. 1981, Ap. J. Letters 251, L69; Bırkınshaw, M., Gull, S.F. and Northover, K.J.E. 1981, M.N.R.A.S. 197, p.571; birkınshaw, M. and Gull, D. F. 1984, M.N.R.A.S. 206, pp. 359-375.

27. Andernach, H., Schallwich, D., Sholomitsk , G.B. and Wielebinski, R. 1983, Ast ron. Ast rophys. 124, pp. 326-330.

28. Bırkinshaw, M., Gull, S.F. and Hardebeck, H. 1984, Nature 309, p. 34.

29. Uson, J. and Wilkinson, D.T. 1984, Bull. Am. Astron. Soc. 16, p. 513.

30. Meyer, S.5. Jeffries, A.D. and Weiss, R. 1983, Ap. J. Letters 271, L1.

\title{
3. Clusters of Galaxies
}

Abell and Corwin (34.160.040) have continued the search of southern clusters on photographs taken with the U.K. Schmidt telescope at Siding Spring. A catalogue is in preparation, it continues the Abell catalogue toward southern declinations. West $(32.160 .019,32.160 .036,34.160 .044)$ has initiated a study of distant southern clusters with automatic identification of clusters and objective evaluation of their properties. Noonan (30.160.006) and Sarazin et al. (31.160.030) have listed clusters with published redshifts. Numerical simulations have demonstrated the presence of bias in observed clusters as difined by Abell (33.160.006), ranked absolute magnitudes of galaxies are distance dependent (33.160.081), some clusters may be due to projection effects (34.160.009).

Kraan-Korteweg has presented a complete sample of bright members of the Virgo cluster and derived the luminosity function and kinematical parameters (30.160.049 31.160.013). Bınggeli, Sandage and Tarenghi (1984) have started a program to study faint galaxies in the Virgo cluster using der Pont 2.5-m reflector. Near infrared photometry of 81 disk galaxies has been done in (34.160.097), structural parameters of 20 galaxies are derived from Fourier and momentum analyses of luminosity profiles (32.160.026). Rocket ultraviolet photometry has been done in (32.160.025). The effective radius and central surface brightness decrease with decreasing luminosity, but there is no difference between 50 and later Hubble type galaxies of same luminosity. Kinematics of the Virgo clusters has been studied also in $(31.160 .072,31.160 .011,33.160 .036,33.160,039)$.

Extensive 21-cm HI observations in Westerbork and Arecibo (30.160.044, $33.160 .003,33.160 .032,33.160 .076,34.160 .005,34.160 .051)$ have demonstrated that galaxies in the Virgo cluster core have smaller HI extent and are HI deficient by a factor 2 to $2.7 \mathrm{in}$ respect to galaxies of the same morphological type outside the core. The HI deficiency depends on the velocity of a galaxy in respect to the cluster suggesting a ram pressure sweeping of the intracluster gas. It is shown that Virgo cluster galaxies are dust poor compared to field galaxies (van den Bergh 1984).

The distribution of radial velocities of galaxies in the vicinity of M87 has demonstrated the presence of background clusters (and probably superclusters) of galaxies at redshifts $V=20200$ and $26200 \mathrm{~km} / \mathrm{s}(31.160 .029,34.157 .034$, Huchra and Brodie 1984).

An extensive radio and optical survey of galaxies in 9 nearby clusters (30.158.060, 30.158.061, 30.158.323, 31.158.324, 32.160.027, Bothun, Schommer, Sullivan 1984) shows that most clusters do not contain gas-deficient spirals. The Coma cluster is the only cluster in the sample exhibiting a large fraction of HI-deficient spirals and having other characteristics consistent with present day spiral-to-So conversion. In general initial conditions of formation and variations in star formation historles have been more important than environmental influences. The key parameter may be the amount of neutral hydrogen remaining after star formation in the bulge has ceased. $21-\mathrm{cm}$ observations of X-ray galaxies in A1367 
show that neutral gas may coexist with the hot coronal $X$-ray gas. Such galaxies are HI deficient (33.160.052). For the Pegasus I cluster also no HI-deficiency has been observed (31.160.047), but in the Hydra I (A1060) and A262 clusters an increase of the HI-deficiency 1 nwards to the cluster center is found $(32.160 .053$, 34.160.012). The effects of viscosity, thermal conduction and turbulence can exceed that due to ram pressure in stripping of clusters of galaxies (31.160.007).

The strıpping of galaxies of gas is most effective in central regions of clusters. The combination of kinematical and HI-deficiency information sugqest that Coma cluster velocity dispersion is isotrope (32.160.001, Pryor, Geller 1984). The total mass of the Coma cluster and its $M / L$ has been estimated from kinematical and $X$-ray data and tidal radii or nearby groups of galaxies. For the Coma cluster mass-to-light radio estimates lie between $100 \mathrm{~h}$ and $400 \mathrm{~h}$ $(30.160 .019, \quad 30.160 .041,31.160 .003,32.160 .016,32.160 .035,34.160 .019$, 34.160.28). Simllar arguments suggest for the Perseus cluster the preference of radial orbits and $(M / L) V=600 \mathrm{~h}(33.160 .080)$.

Radio observations of the Coma $A 1367$ and Perseus clusters have been reported In $(30.160 .011,32.160 .006,33.160 .024)$. Equilibrium distribution of the elements for an isothermal hot plasma in a gravitational potential well suggests that elements with $Z>2$ have abundances proportional to their standard cosmic values but reduced by a factor of $20(30.160 .020)$.

To study photometric properties of galaxies in clusters photographic (31.160.078, 34.160.074), photoelectric (31.160.033) methods and CCD detectors $(33.160 .007,33.160 .019,33.160 .082)$ were used $\mu$ automatic measuring of photographic plates was also used $(33.160 .013)$. CCD photometry of the brightest three galaxies in cores of 83 Abell clusters yields for the dispersion of the absolute magnitudes of first, second, and third brightest galaxy $0.35,0.55$ and 0.65 respectively $(33.160 .082)$. Core radii were determined for 11 rich southern clusters (33.160.037). A new class of clusters that contain a large number of binary gala$x$ les among their brightest members was reported (30.160.045).

The remote cluster of galaxies surrounding 3C 295 has been extensively studied photometrically $(30.160 .046,33.100 .017,34.160 .009)$ and spect roscopically $(32.160 .068,34.160 .001)$. The presence of a large number of blue galaxies is confirmed, but a high fraction of them are likely to be field galaxies. Color distribution of member galaxies is typical for local BM type I clusters. Spectral data demonstrate that 6 blue member galaxies are all unusual, including 3 Seyfert gala$x$ les and 3 galaxies which have bursts of star formation. A high percentage of Seyfert galaxies is expected (Osterbrock 1984), since anong brightest nearby field galaxies the fraction of Seyfert galaxies and optically selected quasars is large.

Photometric and spectral (preferentially CCD) studies of other remote and intermediate redshift clusters $(30.160 .047,30.160 .048,31.160 .016,34.160 .006$, $34.160 .044,34.160 .079)$ demonstrate that a high fraction of blue galaxies in the 3C 295 cluster may be unique. In the $z=0.54$ cluster $0016+16$ virtually all galaxies are intrinsically very red, thus star formation must have ceased in the So galaxies of this cluster more than 9 Gyears ago $(30.160 .047)$. Some blue galaxies found in intermediate redshift clusters correspond to normal Sbc galaxies $(34.160 .079)$.

Internal dynamics of clusters has been studied by conventional spectroscopy (30.160.007, 30.160.021, 30.160.051, 32.160.018, 34.160.008, Dressler 1984, Geller et al. 1984) and Medusa spectroscopy (32.160.074, Ellis et al. 1984). Rough redshifts (accurate to $1800 \mathrm{~km} / \mathrm{s}$ ) can be obtained from objective prism spectra (30.160.002, 33.160.079). Tully-Fisher relation has been used to study the expansion of nearby clusters $(31.160 .046)$. Dynamical modeling suggests that Coma has a static core surrounded by a still collapsing envelope of a radius $10 \mathrm{Mpc}$ $(32.160 .050,34.160 .029)$. Kinematical data have been used to estimate the masses of clusters by varıous methods $(31.160 .028,32.160 .012,32.160 .021)$. The relations between cluster velocity dispersion and other cluster parameters (central density, $X$-ray luminosity, radius, and infrared surface brightness) have been investigated $(30.160 .012,32.160 .002,33.160 .014,34.160 .011)$.

Spectrophotometry can also be done with a CCD camera through narrow filters 
(34.160.065). A number of $X$-ray luminous clusters show bright optical emission from extended filaments $(30.160 .042)$. Very bright emission-line systems seen towards N1275 arise from a high-velocity impact of a foreground galaxy upon the accretion flow of cooling gas in the center of the Perseus cluster (34.160.118). Accretion-driven star formation may explain the unusual blue color of N1275 (33.160.085). Accretion rate into the central galaxy is estimated to be $20-100$ $M_{0} y_{-1}(34.160 .071)$.

Radio observations of clusters at various wavelengths have been continued in Arecibo (30.160.055, 32.160.009), in Westerbork (31.160.006, 31.160.014, 33.160 .023 , 33.160.050, Hanisch 1984a, b) and many other radio observatories (30.160.003, 30.160.005, 30.160.008, 30.160.010, 31.160.036, 32.160.005, $32.160 .015,33.160 .005,33.160 .054,34.160 .022,34.160 .089)$. Clusters possessing radio halo (Coma, Perseus, A2255, A2256, A2319) have a number of striking similarities in their optical, radıo, and $X$-ray properties (32.160.059). These also are due to synchrotron radiation by electrons, leaked out of the cluster radio sources (Valtaoja 1984).

VLA $1465 \mathrm{MHz}$ survey of clusters with redshifts of 0.25 to 0.9 shows strong evolution of the radio luminosity function during this period (32.160.051).

Reviews of X-ray observations of clusters of qalaxies are given by Giacconi (32.160.039) and Forman and Jones (32.160.043). Data reduction of earlier Einstein observations has been continued $(30.160 .013,30.160 .016,30.160 .017,30.160,053$, $31.160 .010,31.160 .041,31.160 .050,31.160 .080,32.160 .007,32.160 .040$, $33.160 .022,33.160 .084,34.160 .017,34.160 .024,34.160 .025,34.160 .082$, Rothenflug et al. 1984, Harris et al. 1984, Henry and Lavery 1984, des Forets et al. 1984). An X-ray survey of clusters at moderate and high redshifts suggests that up to redshift of 0.5 the slope of the luminosity function is constant (32.160.049).

Surface brightness profiles and a comparison with optical features suggests that $X$-ray clusters of galaxies can be devided into two classes : XD have small core radıl and the $X$-ray emission is centered on a central dominant galaxy, $n X D$ clusters have larger core radil and emission is not centered on a stationary galaxy (Jones and Forman 1984). This suggests that the formation of a central galaxy occurs in the early stage of the cluster evolution. Poor clusters with central dominant galaxies have $X$-ray structure similar to rich clusters with dominant galaxies, thus the origin of the dominant galaxy in poor and rich clusters should be similar $(34.160 .070)$.

Clusters of galaxies with dominant CD galaxies have been studied in detail (32.160.058, 34.160.102, 34.160.103, van Breugel, Heckman and Miley 1984, Luqqer 1984). D and CD galaxies lie on peaks in the cluster galaxy distribution, whether or not they are the brightest cluster members (34.160.101). CD qalaxies lie at the kinematical centers of their parent clusters (31.160.015). The stellar velocity dispersion in the individual nuclei of galaxies with multiple nuclei yield $(\mathrm{M} / \mathrm{L})_{\mathrm{B}}=10-20\left(H_{0}=100\right)$, but relative velocities are typically $1000 \mathrm{~km} / \mathrm{s}$ (Merritt 1984a, Tonry 1984). These observations are incompatible with the cannibalism hypothesis. Dynamical friction causes galaxy orbits to decay and produce substantial increase in the number of galaxies which pass near the center without lowering the mean orbital velocity. Thus multiple systems so produced would be transient phenomena. Simulations of the dynamical evolution of clusters of gala$x i e s$ under the influence of galaxy mergers, dynamical friction, two-body relaxa$t i o n$ and tidal stripping have been performed $(31.160 .012,32.160 .042,33.160 .004$, 33.160.072). These studies demonstrate that cluster morphology is probably fixed during the cluster collapse and changes little afterward (Merritt 1984b). Specific globular cluster frequency in central galaxies of nearby clusters ( $V$ irgo and Hydra I) is unusually high, thus central galaxies were very special already at an early stage of the evolution of their parent clusters (33.160.074). The luminosity stripped from galaxies and forming the halo of $c D$ galaxies is proportional to the number of cluster galaxies squared, thus the relative importance of the cD halo is a strong function of the cluster richness (Malumuth and Richstone 1984).

A number of clusters have substructures and secondary maxima in the galaxy distribution $(31.160 .017,31.160 .044,32.160 .003,32.160 .010,32.160 .013$, 
$33.160 .008,34.160 .035,34.160 .045,34.160 .095)$. Some of these clusters may be unbound collections of groups of galaxies, as the Cancer cluster is (33.160.073). Numerical simulations show (McGlynn and Fabian 1984) that two clusters can pass through one another without destroying the individual optical components. In some clusters an encounter may now be underway, as in the Centaurus cluster. Highresolution observations of the cluster A1367 indicate the presence of 8 point sources and 13 extended features associated with cluster galaxies (33.160.010). The structure of clusters with bimodal or clumpy $X$-ray morphology is consistent wath the idea that clusters form by coalescence and merging of subclusters $(32.160 .004)$.

Clusters of galaxies are strongly elongated and nearby clusters point with their long axis to each other $(31.160 .024)$. Galaxies in clusters also show a tendency to point toward their nearest neighbours (34.160.098). Monte-Carlo simulations of the orientations and shapes of galaxies in clusters (31.160.074) demonstrates that phenomenon may be due to the history of the cluster formation via adıabatic scenarıo or to subsequent evolution via tidal forces, the first explanation is more plausible (34.160.094, 34.160.098, Dekel, West and Aarseth 1984).

Sunayev and Zeldovich (34.160.081) published a review on the intergalactic gas in clusters, the microwave background radiation and cosmology. Significant depression in the temperature of the microwave radiation at $10.6 \mathrm{CHz}$ is found only in several clusters (A576, A665, A2218, 0016+16), observations at $20.3 \mathrm{GHz}$ confirm the reality of the effect in most cases (30.160.038, Birkinshaw and Gull 1984). The motion of the cluster can produce two-sided brightness pattern in the microwave background and used to test for transverse motion of clusters (33.160.025). Studies of the intracluster gas have been reported in $(31.160 .001,32.160 .011$, 33.160.069, Nottale 1984). Parameters of the intergalactic medium can be determined by using Lyman-alpha absorbing systems in quasars spectra (33.160.087), diffuse UV-radiation (34.160.015) and by X-ray observations (Forman, Jones and Tucker 1984). Allowed density of the hot intercluster gas is $2 \times 10^{-8} \mathrm{~cm}^{-3}$, allowed temperature is $300 \times 10^{6} \mathrm{~K}$.

Counts of faint galaxies in the vicinity of quasars indicate that quasars are situated in first ranked galaxies of groups and clusters of galaxies (Gehren et al. 1984). Active galaxies have been discovered in clusters A484 and A2534 $(32.160 .023)$.

\section{REFERENCES}

Bıngge 11, B., Sandage, A., Tarenghı, M. : 1984, Astron. J. 89, 64.

Binkinshaw, M., Gull, S.F. : 1984, Mon. Not. R. Astron. Soc. 206, 359.

Bothun, G.D., Schommer, R.A., Sullivan III, W.T. : 1984, Astron. J. 89, 466.

Dekel, A., West, M.J., Aarseth, S.J. : 1984, Astrophys. J. 279, 1.

Dressler, A. : 1984, Astrophys. J. $281,512$.

Ellıs, R.S., Gray, P.M., Carter, D., Godwin, J. : 1984, Mon. Not. R. Astron. Soc. $206,285$.

des Forets, G., Domınguez-Tenreiro, R., Gerbal, D., Mathez, G., Mazure, A., Salvador-Sole, E : 1984, Astrophys. J. 280, 15.

Forman, W., Jones, C., Turner, W. : 1984, Astrophys. J. 277, 19.

Gehren, T., Fried, J. Wehinger, P.A., Wyckoff, S. : 1984, Astrophys. J. $278,11$.

Geller, M.J., Burs, T.C., Bothun, G.D., Huchra, J.P. : 1984, Astron. J. 89, 319.

Hanısh, R.J. : 1984a, Astron. Ast rophys. 139, 276.

Hanush, R.J. : 1984b, Astron. Astrophys. 133, 192.

Harrıs, D.E., Costaın, C.H., Dewdney, P.E. : 1984, Astrophys. J. 280, 532.

Henry, J.P., Lavery, R.J. : 1984, Astrophys. 280, 1.

Huchra, J., Brodıe, J. : 1984, Astrophys. J. 280, 547.

Jones, C., Forman, W. : 1984, Ast rophys. J. 276, 38.

Lugger, P.M., : 1984, Astrophys. J. 278, 51.

Malumuth, E.M., Richstone, D.0.: 1984, Astrophys. J. 276, 413.

McGlynn, T.A., Fabıan, A.C. : 1984, Mon. Not. R. Astron. Soc. 208, 709.

Merritt, D. : 1984a, Astrophys. J. Lett. 280, L5.

Merritt, D. : 1984b, Astrophys. J. 276, 26. 
Nottale, L. : 1984, Mon. Not. R. Astron. Soc. 206, 713.

Osterbrock, D.E.: 1984, Ast rophys. J. Lett. 280, L43.

Pryor, C., Geller, M.J. : 1984, Astrophys. J. 278, 457.

Rothenflug, R., Vigroux, L., Mushorzky, R.F., Holt, S.S. : 1984, Astrophys. J. $279,53$.

Tonry, J.L. : 1984, Ast rophys. J. 279, 13.

Valt aoja, E. : 1984, Astron. Ast rophys. 135, 141.

van Breugel, W., Heckman, T., Mlley, G. : 1984, Astrophys. J. 276, 79.

van der Bergh, S. : 1984, Astron. J. 89, 608.

\section{Galaxy formation \\ (Bernard J.T. Jones)}

This article surveys the literature from July 1981 to June 1984. It is neither possible nor desirable to refer to all papers on the subject, and accordingly only papers that are generally representative of some particular idea are explicitly mentioned. Galaxy Formation by its very nature has considerable overlap with other areas of cosmology such as the anisotropy of the cosmic background radiation, the question of the mass density of the universe, the nature of the large scale clustering, and detalled observations of galaxies. These are all topics covered by other reports to Commission 47 and the reader will therefore find only scant attention paid here to these important subjects.

\section{GENERAL TRENDS DURING THE PERIOD}

These past years have been dominated by two major developments. Firstly there has been the input to Cosmology from high energy physics which has provided us with the "inflationary" models for the early stages of the cosmic expansion and a plethora of exotic particles that might constitute the substantial amounts of "unseen mass" that are thought to pervade the unıverse. Secondly gravitational $\mathrm{N}$-body simulations of cosmic evolution have provided a workbench for testing hypotheses for the formation of galaxies and the clustering process in general. The possible role of galaxy mergers has attracted a lot of attention. Observations of the large scale galaxy distribution have revealed a filamentary structure that is an important target for clustering theories. There has also been substantial input from observations of galaxies that have served to delineate specific chemical and dynamical processes taking place during the birth of a galaxy.

There have been a number of conference proceedings published during this period, these are cited in a separate bibliography. Review articles by Efstathiou and Silk (1983), Matzner (1984), and Peebles (1984c) have appeared. IAU Symposium $N^{\circ} 104$ (see ref $[E]$ ) contains a remarkable varlety of papers on the subject.

The material for this review covers theory and observation as relates to galaxy formation. The evolution that ensues once the final system is assembled and has reached a quasi stationary state w1ll only be touched on here : thus no reference is made to the important issues of the chemical evolution of the disk of our Galaxy, dynamical friction in clusters of the relaxation processes that take place in globular clusters once they have formed. This is inevitably subjective and not without ambiguity, but it is essential in the interests of limiting the length and scope of the article !

\section{EXOTIC PARTICLES AND DARK MATTER}

There is growing (indirect) evidence for the existence of substantial amounts of dark invisible matter in the universe. Indeed, the inflationary model universes demand that the present universe be extremely close to the Einstein de Sitter case and so demand considerable quantities of dark nonbaryonic matter. (See Bean et al., 1983 for the current observational situation). Suggestions as to the nature of this material range from 1 ight $.001 \mathrm{eV}$ axions at the low mass end of the scale, through $100 \mathrm{eV}$ neutrinos to primordial black holes at the other. The invention by high energy physicists of numerous exotic particles that could be of reievance to the earliest moments of the cosmic expansion leads naturally to reconsidering how 